\title{
Experiment research of a rotating cylinder's guidance components on the three-axis turntable
}

\author{
Bo Tang \& Bo Mo \\ School of Aerospace Engineering, Beijing Institute of Technology, Beijing 100081, China
}

\begin{abstract}
Rotating cylinder is a major categories of guidance ammunition now. In order to detect the performance of its guidance components, the hardware-in-the-loop simulation test is necessary. Firstly, a platform whose core is a three-axis turntable is established for the test. Then, two experiments are conducted for the guidance components of a rotating cylinder; one is the high-speed unwinding test of the IMU on strapdown inertial navigation system (SINS) platform, the other is the isolation test for the infrared rate gyro seeker. At last, the performance of the SINS and infrared rate gyro seeker is analyzed bases on the data from the tests.
\end{abstract}

KEYWORD: rotating cylinder; three-axis turntable; SINS; infrared seeker; IMU

\section{INTRODUCTION}

Rotating cylinder is a kind of guidance ammunition which rotate at a certain speed in the air. It has several advantages as follows: less interference from the asymmetric of the force and shape, less damage from the laser weapons, more easily to defense via helix trajectory, etc. [1]. At the same time, the control structure of the rotating cylinder is relatively simple and it is cost-effective, good for mass production and one of the development direction of the guided weapons in the future.

Three-axis turntable which can simulate the changes of the aircraft's attitudes in the air, is a key equipment in the hardware-in-the-loop simulation system on the ground. And the simulation system is very important on the test of the rotating cylinder's guidance components. To verify the performance of the rotating cylinder's guidance components, two experiments are conducted on the three-axis turntable; analysis is made based on the data acquired from the experiments.

\section{THE EXPERIMENT PLATFORM OF THE THREE-AXIS TURNTABLE}

\subsection{The overall architecture of the control system of the three-axis turntable}

The control system of three-axis turntable contains mechanical body, motor control system and the cables. Figure 1 illustrates the details of the system.

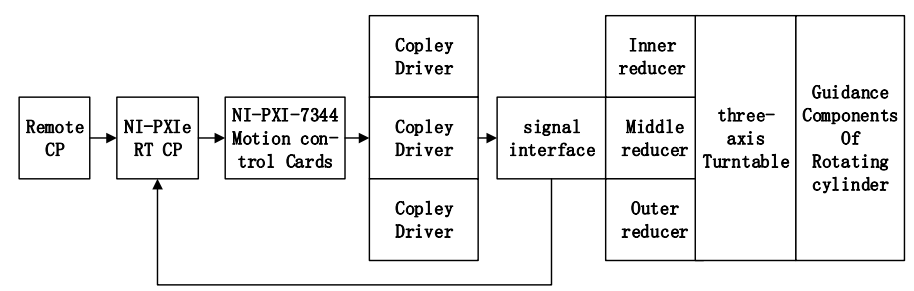

Fig. 1 The control structure of the three-axis turntable

\subsection{The hardware structure of the three-axis turntable}

The three-axis turntable whose structure is $\mathrm{U}-\mathrm{O}-\mathrm{O}$, has three degrees of freedom which are yaw (Z), pitch (Y) and roll (X). And the driver of each frame is the BLDCM (brushless dc motor) which is equipped with precise reducer [2]. The specific structure is shown in Figure 2.

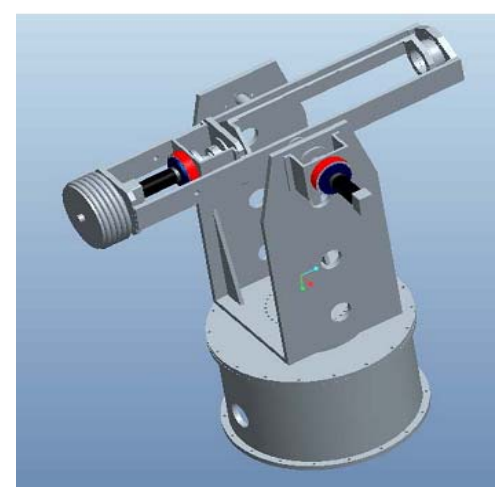

Fig. 2 The mechanical structure of the three-axis turntable 


\subsection{The software system of the three-axis turntable}

The system of the three-axis turntable is used to simulate the Angle motion of aircrafts. So the performance of its motion control card directly determine the smallest step length of the servo system. In order to meet the real-time demand of the semiphysical simulation experiment, a real-time simulation computer (RT) based on the NI PXIe architecture was chosen to control the system, and its remote computer uses the Labview as the development platform [3].

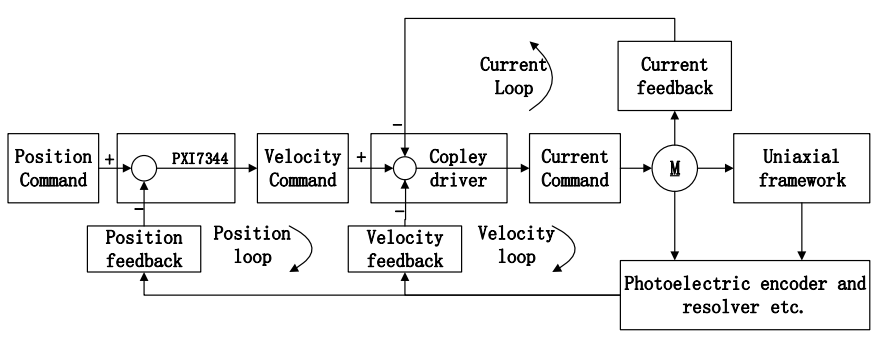

Fig. 3 Single-axis movement control system of the three-axis turntable

The three-axis turntable includes three frameworks which are independent and mutual coupling as well. Uniaxial motion control system which contains an upper computer, a slave computer, a motor driver, a motor reducer, a motor, a framework and the feedback elements is shown in figure 3 .

\section{THE GUIDANCE COMPONENTS OF A CERTAIN ROTATING CYLINDER}

\subsection{The SINS platform containing an unwinding $I M U$}

When a rotating cylinder spins at high speed in the air, the SINS platform and the IMU in it will spins too. If a normal gyro is used in the IMU, there will be a problem of outrange for the gyro $\mathrm{X}$. To solve the problem, a new structure (add an unwinding motor for the IMU) of the SINS platform was designed. Then, the IMU can be considered geosynchronous and work regularly when the unwinding motor spins at the same speed in the opposite direction.

\subsection{Infrared rate gyro seeker}

Seeker, an indispensable part of precision-guided weapons, is the "eyes" for the aircrafts. How does a infrared rate gyro seeker work: First of all, identify the target from the infrared images and measure the deviation of the goal; Secondly, track the target via the deviation information; Finally, calculate the lineof-sight and line-of-sight rate of the target to get the guidance information [4].

\section{THE EXPERIMENTS AND THE RESULTS}

\subsection{Tests for the three-axis turntable}

\subsubsection{The high-speed rolling test for inner frame} When the step instruction of speed loop is 20rps, there is the response curve as shown in figure 4. And figure 4 illustrates that the rising time is $0.75 \mathrm{~s}$ and the steady state error is $0.3 \%$. The performance of the speed control can satisfy the requirements of the experiments later.

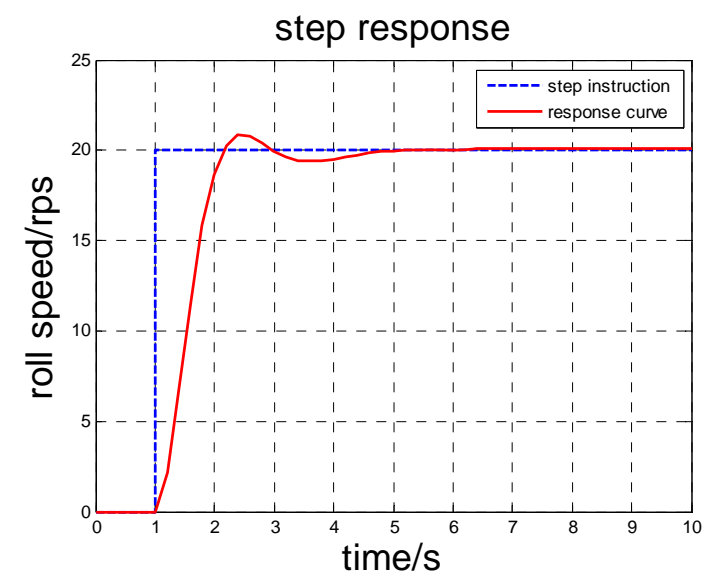

Fig. 4 Response curve of the inner frame at the roll speed of 20rps

\subsubsection{Frequency band test for the three frame}

The position instructions of each frame are sine signals whose amplitude is $2^{\circ}$ and frequency is a range from $1 \mathrm{~Hz}$ to $5 \mathrm{~Hz}$. The results are shown in table 1 , 2, 3.

Table 1 Frequency band characteristic of inner frame

\begin{tabular}{llllll}
\hline Freq.(Hz) & 1 & 2 & 3 & 4 & 5 \\
\hline Phase De. $^{\circ}$ & 3.55 & 5.43 & 7.47 & 8.84 & 14.11 \\
Amp. ratio & 0.995 & 0.9975 & 1.005 & 1.0075 & 1.0125 \\
\hline
\end{tabular}

Table 2 Frequency band characteristic of middle frame

\begin{tabular}{llllll}
\hline Freq.(Hz) & 1 & 2 & 3 & 4 & 5 \\
\hline Phase De. $^{\circ}$ & 1.26 & 3.74 & 7.56 & 8.78 & 12.42 \\
Amp. ratio & 1.003 & 1.027 & 1.002 & 0.988 & 0.992 \\
\hline
\end{tabular}

Table 3 Frequency band characteristic of outer frame

\begin{tabular}{llllll}
\hline Freq.(Hz) & 1 & 2 & 3 & 4 & 5 \\
\hline Phase De. $^{\circ}$ & 2.48 & 3.744 & 7.452 & 9.935 & 15.48 \\
Amp. ratio & 0.929 & 0.980 & 1.010 & 1.030 & 1.060 \\
\hline
\end{tabular}

\subsection{Unwinding experiments of the IMU}

Conditions of the test: fix the SINS platform in the inner frame and let the frame spins at a speed of 8rps.

Then, the results which include angular velocity of $\mathrm{X}$, roll angle and roll speed are shown in figure 5, 6,7 . 


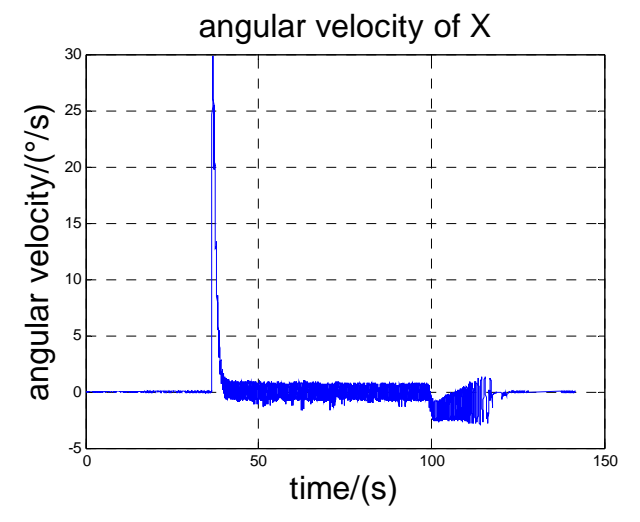

Fig. 5 Roll angular velocity curve of the IMU

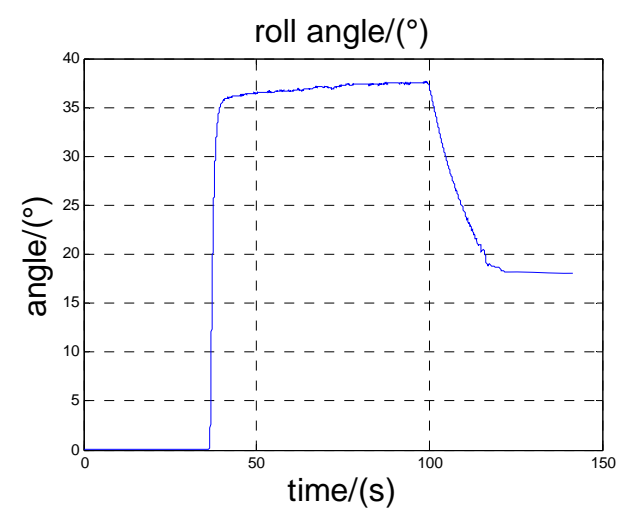

Fig. 6 Roll angle curve of the IMU

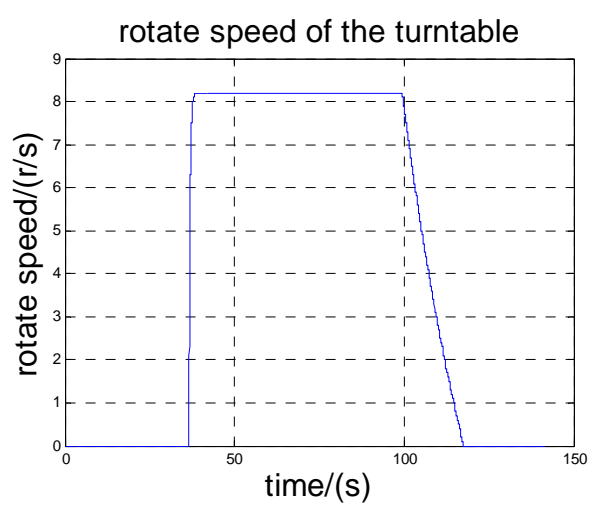

Fig. 7 Rotate speed curve of the turntable

As shown in the figures above, the angular velocity was in almost $0 \%$ s when the inner frame rotates at a high speed. What's more, the maximum of the angular velocity was $30 \%$ shich is less than the range of the gyro. So the experiment could verify that the new structure of the SINS platform is feasible.

\subsection{Isolation test for the infrared rate gyro seeker}

Isolation: The ratio of line-of-sight rate and projectile angular velocity produced by disturbance [5].

Seeker performance metrics: isolation cannot exceed $5 \%$, and the maximum angular velocity produced by disturbance is less than $38 \%$, so line-ofsight rate must be less than $1.9^{\circ} / \mathrm{s}$.
Conditions of the test: Fix the infrared seeker on the end of the middle frame and drive the pitch and yaw axis of the three-axis to simulate the disturbances which both are sinusoidal motions with $2^{\circ}$ and $3 \mathrm{~Hz}$.

Then, the results of the line-of-sight rates are shown in figure $6,7$.

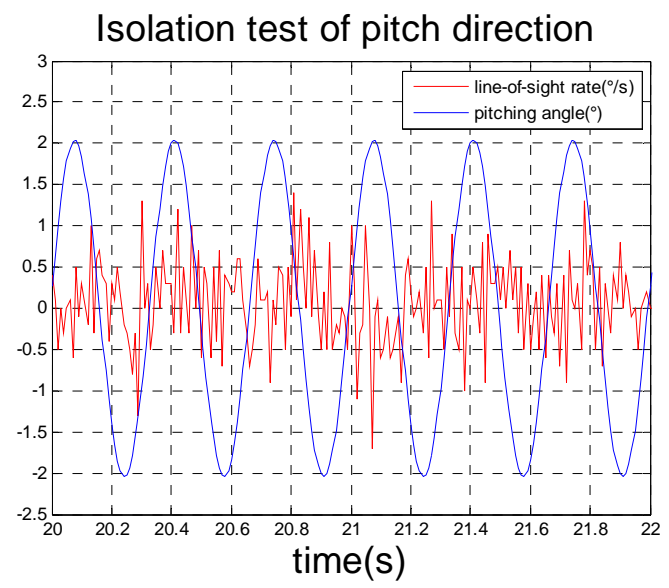

Fig. 8 The curve of the isolation test in pitching direction

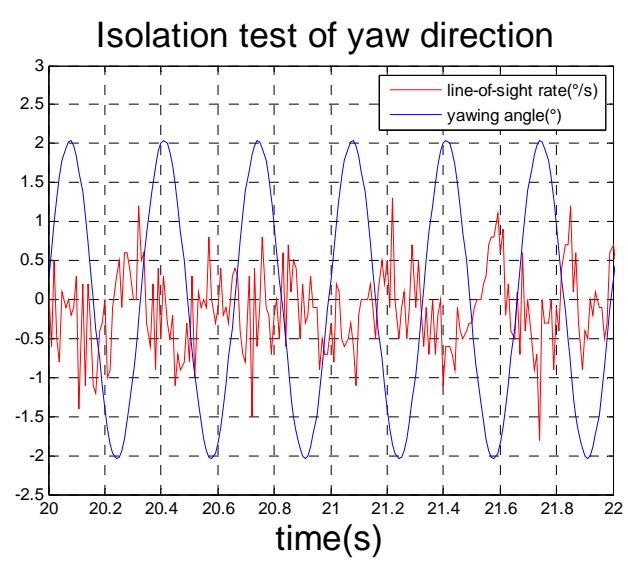

Fig. 9 The curve of the isolation test in yawing direction

As shown in the figures above, the vast majority of line-of-sight rates were less than $1.5 \%$ s both in pitching and yawing direction. Obviously, the results verify that the isolation of the infrared seeker can satisfy the requirements.

\section{SUMMARY}

In this paper, firstly, two experiments are conducted on the three-axis turntable to verify the performance of the two guidance components on a rotating cylinder. One of them was a SINS platform with an unwinding IMU, the other was an infrared rate gyro seeker. The results of the two tests could verify that the new structure of the SINS platform is reasonable and effective and the performance of the infrared seeker could meet the requirements. 


\section{REFERENCES}

[1] Mittal, S.; Kumar, B.. Flow past a rotating cylinder. Journal of Fluid Mechanics, v 476, 303-34, 10 Feb. 2003.

[2] Geng Lei; Ji Xu; Li Hai-yue. Static and dynamic characteristics of three-axis turntable. Modular Machine Tool \& Automatic Manufacturing Technique, n 4, 1-4, 2008

[3] Sanz, D.; Ruiz, M.; Lopez, J.M.; Castro, R.; Vega, J.; Barrera, E. IEEE 1588 clock distribution for FlexRIO devices in PXIe platforms. Fusion Engineering and Design, v 89, n 5, p 652-7, May 2014.

[4] Howser, L.M. Measuring and modeling scan modulation of an infrared seeker. Johns Hopkins APL Technical Digest, v 16, n 1, p 27-33, Jan.-March 1995.

[5] Xu, Ping; Wang, Wei; Lin, De-Fu. Effect of seeker isolation on guidance and control of terminal guided projectile. Dandao Xuebao/Journal of Ballistics, v 24, n 1, p 17-21, March 2012. 